\title{
Theoretical studies of the ground and excited states of the NaLi molecule by the IOTC CASPT 2 method
}

\author{
Marcin Stachowiak $^{1}$ - Maria Barysz ${ }^{1}$ (D) \\ Received: 5 February 2019 / Accepted: 1 April 2019 / Published online: 2 May 2019 \\ (C) The Author(s) 2019
}

\begin{abstract}
Theoretical description of excited states of molecules is still not a routine issue, and searching for methods best suited for this purpose is still an open problem. The molecular calculations are performed using the CASSCF/CASPT2 method for the potential energy curves of the ground and excited states of NaLi molecule. The derived spectroscopic constants are compared with the results of the MR FSCC and PP methods and with the experimental values. The relativistic infinite-order two-component (IOTC) method is used to show its proper convergence to the nonrelativistic limit.
\end{abstract}

Keywords Ground and excited states · Potential energy curves · CASPT2 method - Relativistic infinite order two-component method

\section{Introduction}

The increased interest in cold and ultra-cold techniques of molecular dynamics research and the production of molecules in specific quantum states observed in recent years creates the need for very accurate experimental and theoretical studies of intermolecular interactions of diatomic molecules in ground and excited states. The intensive development of the experimental techniques of recent years has expanded the possibilities of studying such systems and forced the demand for new theoretical research $[1,2]$.

Theoretical calculations of the ground states of molecules and their properties are currently a rather routine task. The exception are the calculations with very high numerical accuracy, and their execution still requires vast knowledge and experience. The situation is different in the case of the excited states.

For many years, research has been conducted on methods that would allow an accurate description of the excited states of molecules. These are studies on single reference methods, for example, such as coupled-cluster theory (CC) [3],

Maria Barysz

teomjb@chem.umk.pl

1 Department of Quantum Chemistry, Faculty of Chemistry, Nicolaus Copernicus University, 7-Gagarina St., 87-100, Toruń, Poland equation of motion (EOM) [4-7], time-dependent density functional theory (TDDFT) $[8,9]$, or multireference (MR) methods such as MR configuration interaction (MRCI) [10], MR coupled-cluster (MRCC, FS-MRCC) [11, 12], MR perturbation theory (MRPT), MR density functional theory (MRDFT), and much more (see the review [12]). This only shows how complicated the problem is.

In recent years, we have witnessed the unique efficiency and accuracy of calculations of excited states energies, properties, and potential energy curves obtained using multireference coupled cluster method in Fock-space (MR FSCC) [13]. Single-reference coupled-cluster methods are based on the single Hartree-Fock determinant and cannot be used to describe the dissociation process. This problem has been solved in the MR FSCC method, in which the reference function of the $N$ electron system can be neutral or ionic with $N \pm 1$ or $N \pm 2$ electron function.

In this work, we will employ one of the most efficient complete active space (CASSCF) method followed by the second-order multireference perturbation (CASPT2) method $[14,15]$. In the CASSCF/CASPT2 method, we do not have any limitations imposed on the reference function. It can be any neutral or ionic structure of the molecule. Additionally, Hartree-Fock molecular orbitals are optimized for the excited states during the CASSCF iteration procedure or they can be replaced by other nonHartree-Fock orbitals. In contrast, in the MR FSCC method, the Hartree-Fock orbitals do not change in the whole computational process. 
The CASSCF/CASPT2 method is known to properly describe the low-lying excited states and their basic spectroscopic parameters. However, its performance for the calculation of the full potential energy curves, is not so obvious due to the intruder states which may occur and the divergence problems.

The NaLi molecule is a frequently chosen example to study the accuracy of various theoretical models because a full range of experimental results for this molecule is available.

The main goal of the present work is to perform quantum-chemical CASSCF/CASPT2 calculations for the $\mathrm{NaLi}$ molecule, determine the full potential energy curves of the ground and a set of excited states, and to calculate the appropriate spectroscopic parameters, such as dissociation energy $D_{e}$; equilibrium distance $R_{e}$; harmonic and anharmonic frequencies $\omega_{e}, \omega_{e} x_{e}$; and adiabatic excitation energies $T_{e}$, and to show that the CASSCF/CASPT2 method can give very good potential energy curves and spectroscopic parameters which correlate well with experimental data. The obtained results will be compared with the results of the MR FSCC and pseudopotential (PP) [16] model (which appeared to work quite well for the studied case).

An additional goal of the work is to check the performance of the relativistic infinite-order two-component (IOTC) method. Relativistic effects are important for heavy elements. The four-component methods work well in atomic calculations; however, they are less effective in molecular calculations. Noteworthy are recently developed four-component relativistic intermediate Hamiltonian Fock-space coupled-cluster method [17] or four-component Dirac-Coulomb(-Gaunt) equation-of-motion coupledcluster theory [18], but their main applications are known for heavy atomic systems or for small molecular structures.

The two-component methods turned out to be a huge success of the relativistic theory, especially in the molecular calculations [19]. The breakthrough was the formulation of two-component methods in which the transformation of a four-component Hamiltonian is accurate. These can be perturbative or one-step unitary transformation methods [19-23]. One of us (MB) has formulated a one-step relativistic two-component method accurate to the infinite order of the fine-structure constant $\alpha=1 / c$, named the IOTC [22, 23].

The NaLi molecule is not relativistic in the sense that the results of nonrelativistic and relativistic calculations should be the same in this case, what simply means that the relativistic effects are small. Showing that the unitary transformation of the relativistic IOTC method converges properly to the nonrelativistic theory is an additional goal of this work. All calculations reported in this paper have been carried out by including relativistic effects and using the
IOTC method. MOLCAS7.3 package of quantum chemistry program modified to include IOTC relativistic Hamiltonian has been used [24, 25].

\section{Computational methodology: NaLi diatomic molecule}

All calculations have been carried out with the CASSCF/CASPT2 method [26, 27]. Calculations have been carried out in $C_{2 v}$ symmetry. The orbital (frozen/inactive/active) subspaces are defined by the number of orbitals in irreducible representations of that group $\left(a_{1}, b_{1}, b_{2}, a_{2}\right)$. The partition of the orbital space used in CASSCF/CASPT2 calculations is then $\left(0.0 .0 .0 / 4.1 .1 .0 / 4.2 .2 .0 ; n_{e l}.\right)$, where $n_{e l}$ is the number of electrons in the active space and $n_{e l}=2$ for NaLi molecule. It means that the active space consists of the $2 s_{\mathrm{Li}}, 2 p_{\mathrm{Li}}$, $3 s_{\mathrm{Na}}$, and $3 p_{\mathrm{Na}}$ valence orbitals.

The active orbital subspaces used in this study are relatively small but are sufficient to calculate the static electronic correlation at the CASSCF theory level. The dynamic electronic correlation is taken into account at the CASPT2 level of theory and the electron correlation treatment is extended by the $1 s_{\mathrm{Li}}, 1 s_{\mathrm{Na}}, 2 s_{\mathrm{Na}}$, and $2 p_{\mathrm{Na}}$ inactive orbitals.

For the calculations of NaLi molecule, we employ the POLDK and two relativistic atomic natural orbitals (ANORCC) Gaussian basis sets of the following structures:

POLDK basis set: $\mathrm{Na}[13 s .10 p .4 d / 7 s .4 p .2 d]$; Li[10s.6p.4d/5s.3p.2d] [29].

ANO-RCC VTZP basis set:

$\mathrm{Na}[17 s .12 p .5 d .4 f / 5 s .4 p .2 d, 1 f]$;

$\operatorname{Li}[14 s .9 p .4 d, 3 f / 4 s .3 p .2 d, 1 f][28]$.

ANO-RCC-large basis set:

$\mathrm{Na}[17 s .12 p .5 d .4 f / 9 s .8 p .5 d .4 f]$;

$\mathrm{Li}[14 s .9 p .4 d, 3 f, 1 g / 8 s .7 p .4 d, 2 f, 1 g][28]$.

where the notation is [uncontracted/contracted]. The POLDK basis set has been originally designed to calculate the electric properties of molecules, but it has worked quite well for the excited states. The MR FSCC calculations used the nonrelativistic POL basis set [13]; therefore, we have chosen the relativistic equivalent of this base, POLDK to our calculations.

Our studies on the NaLi molecule begin with the analysis of the quality of the selected atomic base. The ionization potentials and electron affinity of $\mathrm{Na}$ and $\mathrm{Li}$ atoms were calculated. Atomic calculations have been carried out in $C_{i}$ symmetry. The irreducible representations of this group are $a_{g}$ and $a_{u}$. The partition of the orbital space used in the atomic CASSCF/CASPT2 calculations is then (0.0/1.0/7.6; 
Table 1 Ionization potential (IP) and electron affinity (EA) calculated in the relativistic IOTC CASSCF/CASPT2 method using POLDK and ANO-RCC: VTZP and large basis sets

\begin{tabular}{llllll}
\hline & Basis set & VTZP & POLDK & ANO-large & exp. \\
\hline $\mathrm{Li}$ & IP(eV) & 5.34 & 5.32 & 5.34 & $5.39^{\mathrm{a}}$ \\
& EA(eV) & 0.414 & 0.575 & 0.575 & $0.618^{\mathrm{b}}$ \\
$\mathrm{Na}$ & IP(eV) & 5.055 & 5.091 & 5.111 & $5.139^{\mathrm{a}}$ \\
& EA(eV) & 0.313 & 0.488 & 0.461 & $0.547^{\mathrm{b}}$ \\
\hline
\end{tabular}

${ }^{\mathrm{a}}$ https://chemglobe.org/ptoe-/

${ }^{\mathrm{b}}$ https://periodictable.com/Properties/A/

$\left.1_{e l}\right)$ for lithium (i.e., $2 s, 2 p, 3 s, 3 p$, and $3 d$ active orbitals), and $\left(0.0 / 2.3 / 7.6 ; 1_{e l}\right)$ for sodium (i.e., $3 s, 3 p, 3 d 4 s$, and $4 p$ active orbitals). The relevant data is collected in Table 1 . We see that all three basis give good atomic ionization potentials. Electron affinities are also good, but the results of ANO-RCC VTZP basis seem to be the poorest, as the calculated VTZP values of the electron affinity differ from the experimental value by $0.204 \mathrm{eV}$ for $\mathrm{Li}$ and $0.234 \mathrm{eV}$ for $\mathrm{Na}$ atoms. In conclusion, the POLDK and ANO-RCClarge basis should be best suited to our research. However, for comparison, we will also carry out calculations using ANO-RCC VTZP basis.

\section{Results and discussion}

In Fig. 1, we present potential energy curves for the ground state and seven excited states of the NaLi molecule. The potential energy curves shown in Fig. 1 represent molecule dissociating to $\mathrm{Li}(2 s)+\mathrm{Na}(3 s), \operatorname{Li}(2 p)+\mathrm{Na}(3 s)$, and $\mathrm{Li}(2 s)+\mathrm{Na}(3 p)$ dissociation channels. The large number of calculated energy points and the lack of the intruder states allowed to receive curves which are smooth and of the good quality.

Comparison of the curves obtained by the IOTC CASSCF/CASPT2 method with the curves obtained by the MR FSCC [13] and PP [16] methods shows qualitative agreement and identical interpretation of the obtained states in all three methods. The only exceptions are $3{ }^{1} \Sigma^{+}$and $2^{1} \Pi$ states, discussed later in this work.

In Tables 2, 3, and 4, we present calculated spectroscopic parameters for the studied states compared with the results obtained with the MR FSCC and PP methods. Experimental results are given where possible.

The ground state of the NaLi molecule is, as it has been expected, the $X^{1} \Sigma^{+}$state and it dissociates to the $L i(2 s)+$
Fig. 1 Potential energy curves for the ground and excited states of the NaLi molecule with the IOTC CASSCF/CASPT2 method for $\mathrm{Li}(2 s)+\mathrm{Na}(3 s)$, $\mathrm{Li}(2 p)+\mathrm{Na}(3 s)$, and $\mathrm{Li}(2 s)+\mathrm{Na}(3 p)$ dissociation limits in ANO-RCC-large basis set

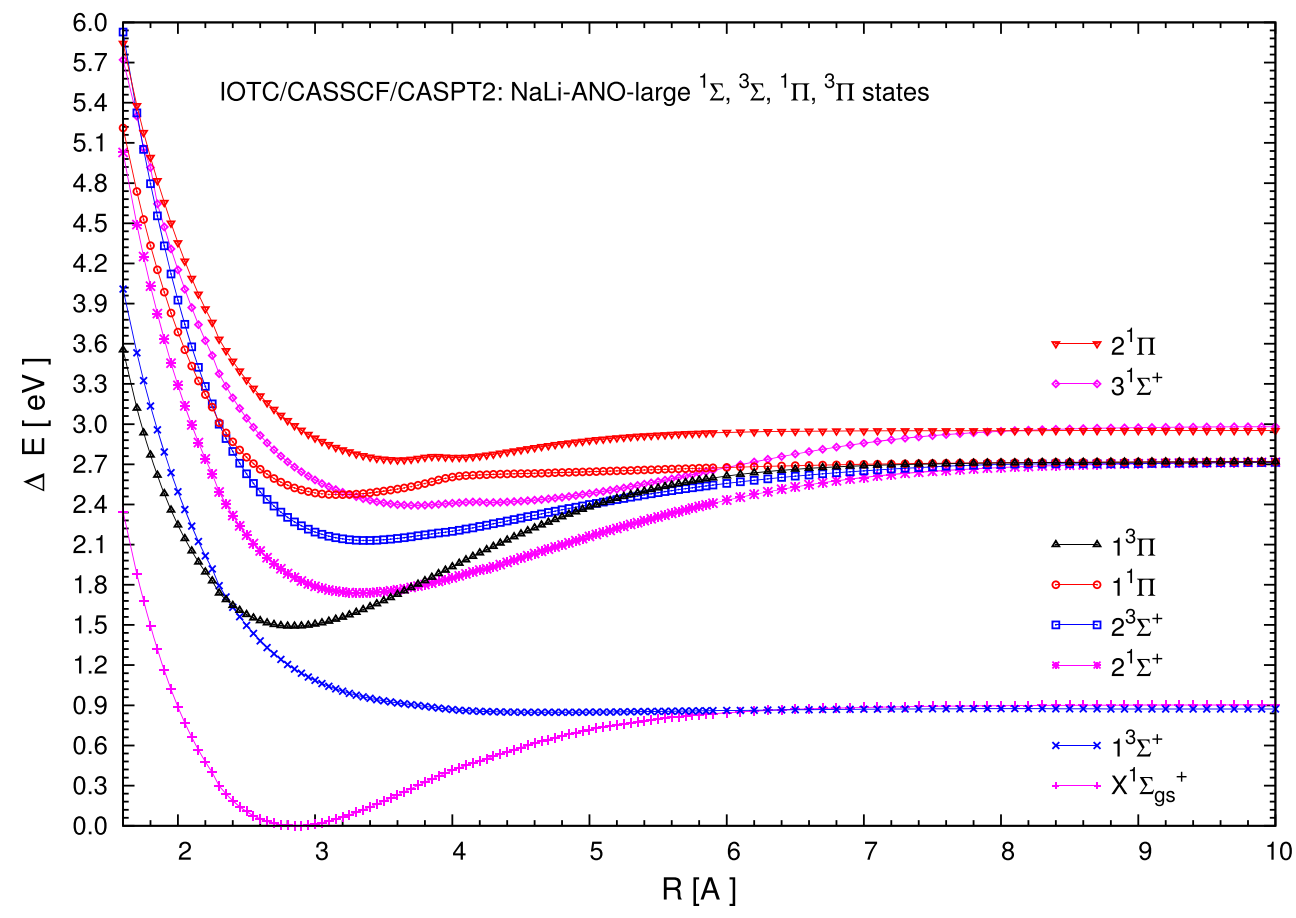


Table 2 Spectroscopic constants $\left(R_{e}\right.$ in $\AA ; D_{e}, T_{e}$ in eV; $\omega_{e}, \omega_{e} x_{e}$ in $\left.\mathrm{cm}^{-1}\right)$ for the NaLi molecule in the relativistic IOTC CASSCF/CASPT2 method using POLDK and ANO-RCC: VTZP and large basis sets. $X^{1} \Sigma^{+}$and $1^{3} \Sigma^{+}$states

\begin{tabular}{|c|c|c|c|c|c|c|}
\hline \multicolumn{7}{|c|}{$\mathrm{Li}(2 s)+\mathrm{Na}(3 s)$ dissociation limit } \\
\hline \multicolumn{7}{|l|}{$X^{1} \Sigma^{+}$} \\
\hline$R_{e}$ & 2.843 & 2.878 & 2.854 & 2.864 & 2.873 & 2.885 \\
\hline$D_{e}$ & 0.8926 & 0.8268 & 0.9052 & 0.901 & 0.8746 & 0.8763 \\
\hline$\omega_{e}$ & 262.4 & 249.6 & 261.1 & 252 & 256.52 & 256.80 \\
\hline$\omega_{e} x_{e}$ & 1.5 & 1.5 & 1.4 & - & 1.72 & 1.66 \\
\hline$T_{e}$ & 0.0 & 0.0 & 0.0 & 0.0 & 0.0 & 0.0 \\
\hline \multicolumn{7}{|l|}{$1^{3} \Sigma^{+}$} \\
\hline$R_{e}$ & 4.495 & 4.738 & 4.713 & 4.494 & 4.720 & - \\
\hline$D_{e}$ & 0.0375 & 0.0327 & 0.0286 & 0.052 & 0.0272 & - \\
\hline$\omega_{e}$ & 49.5 & 50.4 & 41.6 & 46 & 40.56 & - \\
\hline$\omega_{e} x_{e}$ & 2.3 & 1.8 & 0.4 & - & 1.91 & - \\
\hline$T_{e}$ & 0.832 & 0.774 & 0.847 & 0.850 & 0.847 & - \\
\hline
\end{tabular}

${ }^{\mathrm{a}}$ Reference [13]. ${ }^{\mathrm{b}}$ Reference [16]

Table 3 Spectroscopic constants $\left(R_{e}\right.$ in $\AA ; D_{e}, T_{e}$ in eV; $\omega_{e}, \omega_{e} x_{e}$ in $\left.\mathrm{cm}^{-1}\right)$ for the NaLi molecule in the relativistic IOTC CASSCF/CASPT2 method using POLDK and ANO-RCC: VTZP and large basis sets. $2^{1} \Sigma^{+}, 2^{3} \Sigma^{+}, 1^{1} \Pi, 1^{3} \Pi$ states

\begin{tabular}{|c|c|c|c|c|c|c|}
\hline $\begin{array}{l}\text { Basis set } \\
\operatorname{Li}(2 p)+1\end{array}$ & $\begin{array}{l}\text { VTZP } \\
\text { ociation }\end{array}$ & POLDK & ANO-large & MR FSCC ${ }^{a}$ & $\mathrm{PP}^{\mathrm{b}}$ & exp. ${ }^{b}$ \\
\hline \multicolumn{7}{|l|}{$2^{1} \Sigma^{+}$} \\
\hline$R_{e}$ & 3.561 & 3.369 & 3.327 & 3.370 & 3.360 & - \\
\hline$D_{e}$ & 0.9883 & 0.9933 & 0.9801 & 0.987 & 0.9744 & 0.964 \\
\hline$\omega_{e}$ & 183.0 & 185.0 & 194.9 & 182 & 189.56 & 191 \\
\hline$\omega_{e} x_{e}$ & 44.9 & 0.1 & 1.5 & - & 1.7 & - \\
\hline $\begin{array}{l}T_{e} \\
2^{3} \Sigma^{+}\end{array}$ & 1.738 & 1.700 & 1.739 & 1.754 & 1.748 & 1.760 \\
\hline$R_{e}$ & 3.489 & 3.429 & 3.371 & 3.454 & 3.440 & - \\
\hline$D_{e}$ & 0.5714 & 0.5594 & 0.5958 & 0.610 & 0.5949 & - \\
\hline$\omega_{e}$ & 163.8 & 179.5 & 186.7 & 162 & 173.08 & - \\
\hline$\omega_{e} x_{e}$ & 1.5 & 1.1 & 2.9 & - & 1.60 & - \\
\hline \multicolumn{7}{|l|}{$1^{1} \Pi$} \\
\hline$R_{e}$ & 3.179 & 3.252 & 3.180 & 3.212 & 3.228 & 3.119 \\
\hline$D_{e}$ & 0.2044 & 0.1848 & 0.2508 & 0.244 & 0.2134 & 0.2368 \\
\hline$\omega_{e}$ & 182.8 & 171.6 & 191.3 & 167 & 177.75 & 209.63 \\
\hline$\omega_{e} x_{e}$ & 2.0 & 5.4 & 3.3 & - & 4.06 & 9.979 \\
\hline \multicolumn{7}{|l|}{$1^{3} \Pi$} \\
\hline$R_{e}$ & 2.830 & 2.860 & 2.837 & 2.830 & 2.841 & - \\
\hline$D_{e}$ & 1.2509 & 1.1877 & 1.2331 & 1.242 & 1.2031 & - \\
\hline$\omega_{e}$ & 252.4 & 245.8 & 252.4 & 248 & 250.99 & - \\
\hline$\omega_{e} x_{e}$ & 1.2 & 1.2 & 1.1 & - & 1.29 & - \\
\hline$T_{e}$ & 1.461 & 1.459 & 1.490 & 1.499 & 1.519 & - \\
\hline
\end{tabular}

${ }^{\mathrm{a}}$ Reference [13], ${ }^{\mathrm{b}}$ Reference [16] 
Table 4 Spectroscopic constants $\left(R_{e}\right.$ in $\AA ; D_{e}, T_{e}$ in eV; $\omega_{e}, \omega_{e} x_{e}$ in $\left.\mathrm{cm}^{-1}\right)$ for the NaLi molecule in the relativistic IOTC CASSCF/CASPT2 method using POLDK and ANO-RCC: VDZP, VTZP and large basis sets. $3^{1} \Sigma^{+}$and $2^{1} \Pi$ states

\begin{tabular}{|c|c|c|c|c|c|c|}
\hline \multicolumn{7}{|c|}{$\mathrm{Li}(2 s)+\mathrm{Na}(3 p)$ dissociation limit } \\
\hline \multicolumn{7}{|l|}{$3^{1} \Sigma^{+}$} \\
\hline$R_{e}$ & 4.081 & - & - & 4.102 & 4.164 & - \\
\hline$D_{e}$ & 0.5241 & - & - & 0.549 & 0.5390 & 0.6725 \\
\hline$\omega_{e}$ & 114.6 & - & - & 110 & 106.20 & 160 \\
\hline$\omega_{e} x_{e}$ & 0.07 & - & - & - & -0.39 & 1.1 \\
\hline \multicolumn{7}{|l|}{$2^{1} \Pi$} \\
\hline$R_{e}$ & 3.875 & - & - & 3.738 & 3.725 & - \\
\hline$D_{e}$ & 0.1598 & - & - & 0.234 & 0.2135 & 0.2185 \\
\hline$\omega_{e}$ & 169.6 & - & - & 149 & 155.25 & 165 \\
\hline$\omega_{e} x_{e}$ & - & - & - & - & 1.97 & 3.1 \\
\hline$T_{e}$ & 2.750 & - & - & 2.752 & 2.765 & 2.760 \\
\hline
\end{tabular}

${ }^{\mathrm{a}}$ Reference [13], ${ }^{\mathrm{b}}$ Reference [16]

$N a(3 s)$ dissociation channel. The IOTC CASSCF/CASPT2 equilibrium distance, dissociation energy, harmonic frequency, and the first anharmonicity corrections calculated in the ANO-RCC-large basis are $R_{e}=2.854 \AA, D_{e}=$ $0.905 \mathrm{eV}, \omega_{e}=261 \mathrm{~cm}^{-1}$, and $\omega_{e} x_{e}=1.4 \mathrm{~cm}^{-1}$. All parameters agree well with experimental values and with the results of MR FSCC and PP methods. The CASPT2 results are very good but the the compatibility of calculated PP parameters with experimental values is even better. We observe such good PP consistency for most of calculated excited states.

The studied excited states are divided into three groups, the $1^{3} \Sigma^{+}$which dissociate to the same dissociation limit as the ground state, the $2^{1} \Sigma^{+}, 2^{3} \Sigma^{+}, 1^{1} \Pi$, and $1^{3} \Pi$ states which dissociate to the $\operatorname{Li}(2 p)+\mathrm{Na}(3 s)$ channel, the $3^{1} \Sigma^{+}$ and $2^{1} \Pi$ states, which dissociate to the $\mathrm{Li}(2 s)+\mathrm{Na}(3 p)$ channel.

The first excited state is the $1^{3} \Sigma^{+}$state. Experimental data for this state is not available. One can see (Table 2) that the spectroscopic parameters calculated by CASPT2 method agree very well with the parameters calculated using the PP method. The corresponding MR FSCC parameters differ significantly from the PP and CASPT2 values. The MR FSCC equilibrium distance $R_{e}$ is longer than the PP and CASPT 2 values by about $0.2 \AA$, the MR FSCC dissociation energy $0.052 \mathrm{eV}$ is almost twice as big as calculated by CASPT2 and PP methods. However, one must pay attention to the fact that the dissociation energy for $1^{3} \Sigma^{+}$state is very small and is 0.0286 and $0.0272 \mathrm{eV}$ respectively for the CASPT2 and PP methods. The harmonic frequencies for the MS FSCC, CASPT2, and PP methods are respectively $42 \mathrm{~cm}^{-1}, 46 \mathrm{~cm}^{-1}$, and $41 \mathrm{~cm}^{-1}$, and they are all of the similar accuracy. The CASPT2 and PP $\omega_{e} x_{e}$ are 0.4 and $1.91 \mathrm{~cm}^{-1}$.

The second set of investigated excited states include $2^{1} \Sigma^{+}, 2^{3} \Sigma^{+}, 1^{1} \Pi$, and $1^{3} \Pi$ states that dissociate to $\mathrm{Li}(2 p)+\mathrm{Na}(3 s)$ channel. The results of calculations are presented in Table 3 . The analysis of calculated $R_{e}, D_{e}$, and $\omega_{e}$ parameters shows that all three CASPT2, MR FSCC, and PP methods give similar results which agree very well with experimental data, where available. The biggest differences can be observed for the harmonic frequencies $\omega_{e}$ obtained in the FSCC method. It seems that the value of this parameter is too small for almost all four calculated excited states. This difference is particularly visible for $1^{1} \Pi$ state for which the $\omega_{e}$ values calculated in the CASPT2, MR FSCC, and PP methods are $191 \mathrm{~cm}^{-1}, 167 \mathrm{~cm}^{-1}$, and $178 \mathrm{~cm}^{-1}$. The corresponding experimental value is $210 \mathrm{~cm}^{-1}$. The POL basis used in MR FSCC calculations could be too small for these studied states.

The calculated CASPT2 anharmonic frequencies $\omega_{e} x_{e}$ are quite good and correlate well with PP values. The only exception is $1^{1} \Pi$ but both CASPT2 and PP values differ from the experimental values.

The most difficult to interpret are highest $3^{1} \Sigma^{+}$and $2^{1} \Pi$ excited states. The ANO-RCC-large CASPT 2 potential energy curves shown in Fig. 1 are smooth and of good quality. However, a detailed analysis of the curves (see Fig. 2) shows two minima on both curves, which we do not observe in earlier MR FSCC and PP studies. Similarly, shaped curves were obtained from POLDK CASPT2 calculations (which are not presented here). On the other hand, no double minima appear on the calculated VTZP CASPT2 curves (Fig. 3). 
Fig. 2 Potential energy curves for the $3^{1} \Sigma^{+}$and $2^{1} \Pi$ excited states of the NaLi molecule with the IOTC CASSCF/CASPT2 method in the ANO-RCC-large basis set

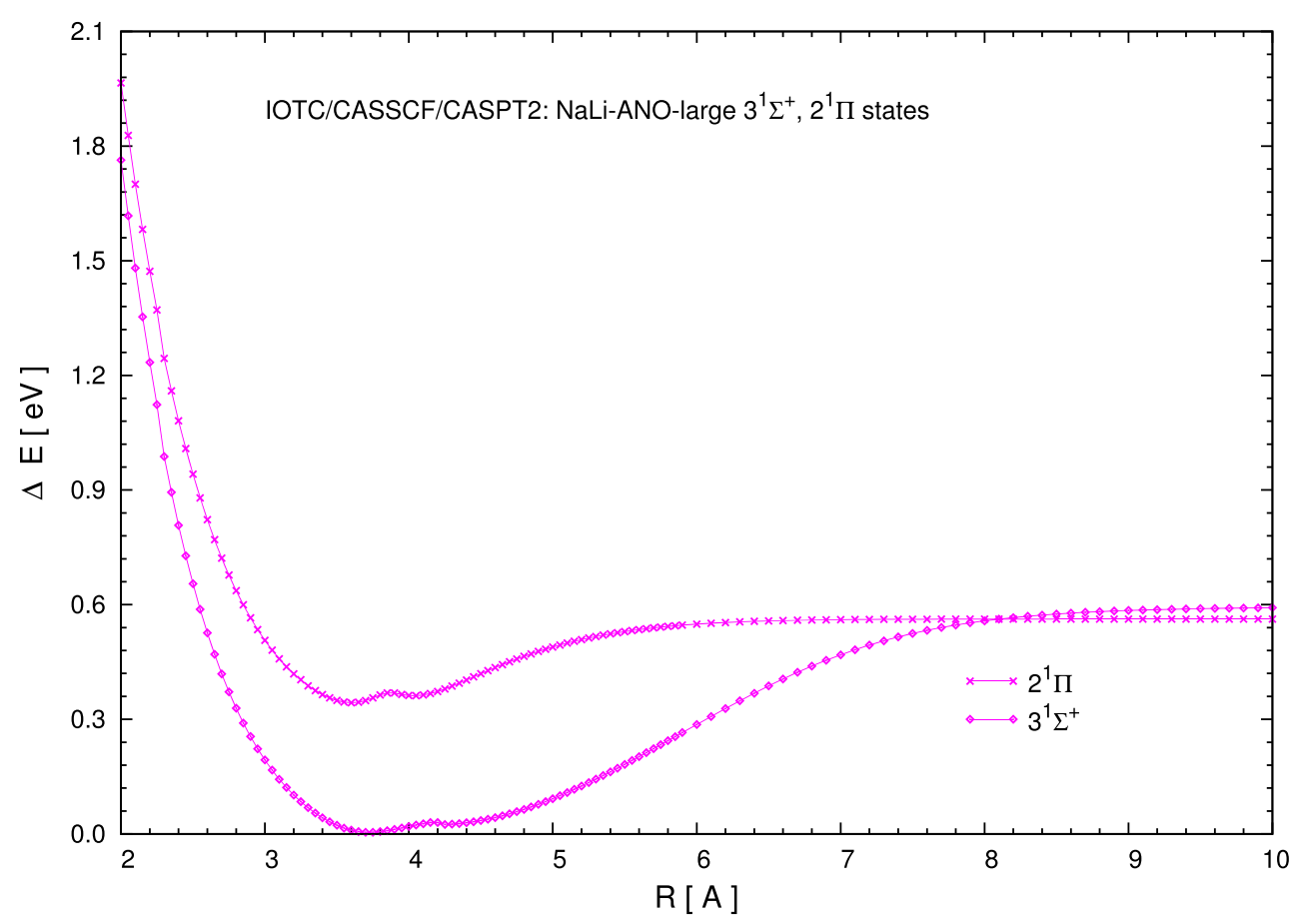

The VIBROT program we are using (included in the MOLCAS 7.3 package) for vibration and rotation analysis has not been developed to analyze multiple minima curves, and we could not calculate the corresponding spectroscopic parameters in POLDK and ANO-RCC-large basis. As it could be expected, we observe fairly good agreement
Fig. 3 Potential energy curves for the $3^{1} \Sigma^{+}$and $2^{1} \Pi$ excited states of the NaLi molecule with the IOTC CASSCF/CASPT2 method in the ANO-RCC VTZP basis set

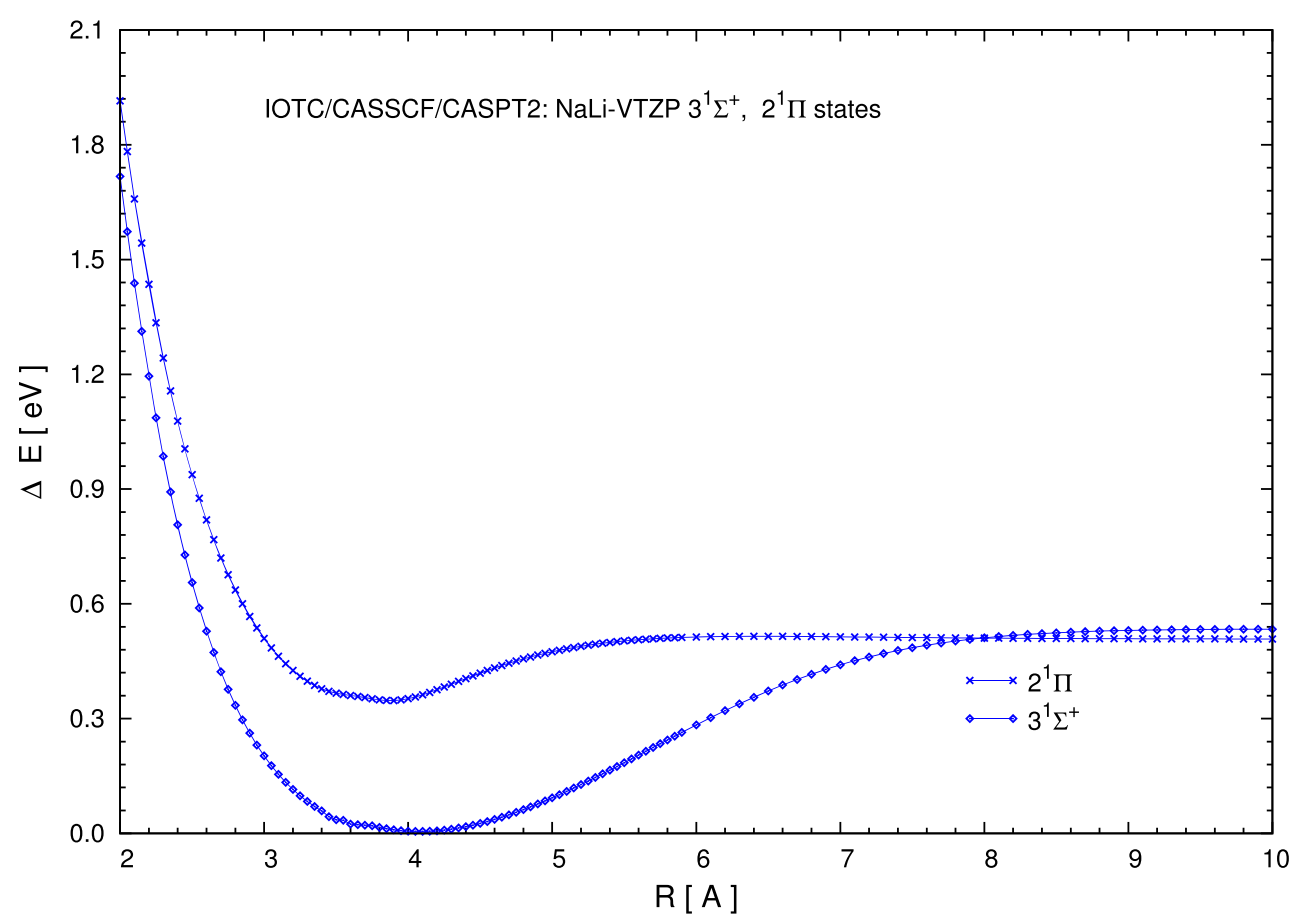


Table 5 Energies (in a.u.) at the dissociation limit in IOTC/CASSCF/CASPT2 method

\begin{tabular}{|c|c|c|c|}
\hline Basis set & $\begin{array}{l}E_{14.00} \text { (a.u.) } \\
\text { VTZP }\end{array}$ & POLDK & ANO-large \\
\hline \multicolumn{4}{|c|}{$\mathrm{Li}(2 s)+\mathrm{Na}(3 s)$ dissociation limit } \\
\hline$X^{1} \Sigma^{+}$ & -169.7327574180 & -169.5797600724 & -169.7920205121 \\
\hline $1^{3} \Sigma^{+}$ & -169.7335871371 & -169.5805695902 & -169.7931181333 \\
\hline \multicolumn{4}{|c|}{$\mathrm{Li}(2 p)+\mathrm{Na}(3 s)$ dissociation limit } \\
\hline $2^{1} \Sigma^{+}$ & -169.6658433034 & -169.5128031336 & -169.7251268895 \\
\hline $2^{3} \Sigma^{+}$ & -169.6658821590 & -169.5128592820 & -169.7251845283 \\
\hline $1^{1} \Pi$ & -169.6658944431 & -169.5128958458 & -169.7252364928 \\
\hline $1^{3} \Pi$ & -169.6658842654 & -169.5128946348 & -169.7252321062 \\
\hline \multicolumn{4}{|c|}{$\mathrm{Li}(2 s)+\mathrm{Na}(3 p)$ dissociation limit } \\
\hline $3^{1} \Sigma^{+}$ & -169.6576823777 & -169.5032993066 & -169.7156140827 \\
\hline $2^{1} \Pi$ & -169.6585608387 & -169.5041621293 & -169.7167808612 \\
\hline
\end{tabular}

between the calculated parameters in VTZP CASPT2, MR FSCC, and PP basis sets (see Table 4); however, the quality of these results is not certain.

An additional parameter, which has been calculated for all studied states is adiabatic excitation energy $T_{e}$ (see Tables 2, 3, and 4), which characterize the electronic spectra of NaLi molecule. The CASPT2 excitation energies $T_{e}$ agree very well with both MR FSCC and PP values and the corresponding experimental data.

Finally, we present the energies of the electronic states calculated at the dissociation limits of the $\mathrm{NaLi}$ molecule (Table 5). The corresponding excitation energies are compared with the sum of atomic energies (Table 6). These results confirm the correct behavior of the CASPT2 method and its size-consistency feature.

\section{Convergence of the relativistic IOTC method to the nonrelativistic limit}

One of the final goals of the present work was to analyze the accuracy of the relativistic infinite-order two-component
IOTC method. The NaLi molecule is composed of light lithium and sodium atoms and the relativistic effects should be very small, and even negligible, if we are interested in the chemical accuracy of the calculations. Nature is always relativistic. The relativistic method should be general. It should give correct results for strongly relativistic systems but it should also smoothly converge to the nonrelativistic equations when the relativistic effect is negligible.

The correctness of the IOTC method has been checked by performing relativistic IOTC CASSCF/CASPT2 and nonrelativistic NR CASSCF/CASPT2 calculations in the uncontracted POLDK basis for the ground and excited states. The calculated spectroscopic parameters are presented in Tables 7 and 8. The obtained results are almost identical in both relativistic and non-relativistic calculations. This means that the IOTC method correctly converges to the non-relativistic limit when the relativistic effects disappear or are very small, as in the case of the $X^{1} \Sigma^{+}$ground state for which we observe relativistic bond length contraction $2.855 \AA$ (IOTC) vs $2.887 \AA$ (NR) (see Table 7).

Table 6 Excitation energies (in eV) calculated at the dissociation limit of NaLi molecule compared with the atomic energies

\begin{tabular}{llll}
\hline Basis set/method & VTZP & POLDK & ANO-large \\
\hline & $\operatorname{Li}(2 s) \rightarrow \operatorname{Li}(2 p)$ & & \\
Molecular & 1.821 & 1.822 & 1.820 \\
Atomic & 1.841 & 1.828 & 1.841 \\
$E(\mathrm{eV})$ & $\mathrm{Na}(3 s) \rightarrow \mathrm{Na}(3 p)$ & & \\
Molecular & 2.043 & 2.081 & 2.080 \\
Atomic & 2.065 & 2.086 & 2.094 \\
\hline
\end{tabular}

ahttps://www.nist.gov/pml/handbook-basic-atomic-spectroscopic-data and select "Element Name" and "Energy Levels" 
Table 7 Spectroscopic constants $\left(R_{e}\right.$ in $\AA ; D_{e}, T_{e}$ in eV; $\omega_{e}, \omega_{e} x_{e}$ in $\left.\mathrm{cm}^{-1}\right)$ for the NaLi Molecule in the relativistic IOTC CASSCF/CASPT2 and non-relativistic (NR) CASSCF/CASPT2 method using uncontracted POLDK basis set. $X^{1} \Sigma^{+}, 1^{3} \Sigma^{+}, 2^{1} \Sigma^{+}, 2^{3} \Sigma^{+}$states

\begin{tabular}{|c|c|c|c|}
\hline \multirow[t]{2}{*}{ State } & & \multicolumn{2}{|l|}{ Method } \\
\hline & & IOTC CASSCF/CASPT2 & NR CASSCF/CASPT2 \\
\hline \multirow[t]{5}{*}{$X^{1} \Sigma^{+}$} & $R_{e}(\AA)$ & 2.855 & 2.887 \\
\hline & $D_{e}(\mathrm{eV})$ & 0.827 & 0.832 \\
\hline & $\omega_{e}\left(\mathrm{~cm}^{-1}\right)$ & 253.5 & 253.4 \\
\hline & $\omega_{e} x_{e}\left(\mathrm{~cm}^{-1}\right)$ & 1.6 & 1.6 \\
\hline & $T_{e}(\mathrm{eV})$ & 0.0 & 0.0 \\
\hline \multirow[t]{5}{*}{$1^{3} \Sigma^{+}$} & $R_{e}(\AA)$ & 5.011 & 5.007 \\
\hline & $D_{e}(\mathrm{eV})$ & 0.016 & 0.016 \\
\hline & $\omega_{e}\left(\mathrm{~cm}^{-1}\right)$ & 38.4 & 38.6 \\
\hline & $\omega_{e} x_{e}\left(\mathrm{~cm}^{-1}\right)$ & 1.8 & 1.9 \\
\hline & $T_{e}(\mathrm{eV})$ & 0.784 & 0.784 \\
\hline \multirow[t]{5}{*}{$2^{1} \Sigma^{+}$} & $R_{e}(\AA)$ & 3.376 & 3.378 \\
\hline & $D_{e}(\mathrm{eV})$ & 0.956 & 0.958 \\
\hline & $\omega_{e}\left(\mathrm{~cm}^{-1}\right)$ & 186.8 & 187.4 \\
\hline & $\omega_{e} x_{e}\left(\mathrm{~cm}^{-1}\right)$ & 0.8 & 1.0 \\
\hline & $T_{e}(\mathrm{eV})$ & 1.702 & 1.699 \\
\hline \multirow[t]{5}{*}{$2^{3} \Sigma^{+}$} & $R_{e}(\AA)$ & 3.419 & 3.420 \\
\hline & $D_{e}(\mathrm{eV})$ & 0.558 & 0.561 \\
\hline & $\omega_{e}\left(\mathrm{~cm}^{-1}\right)$ & 183.6 & 183.6 \\
\hline & $\omega_{e} x_{e}\left(\mathrm{~cm}^{-1}\right)$ & 1.3 & 1.6 \\
\hline & $T_{e}(\mathrm{eV})$ & 2.099 & 2.095 \\
\hline
\end{tabular}

\section{Summary}

The spectroscopic parameters and potential energy curves of the NaLi molecule in the gas phase were obtained using the IOTC CASSCF/CASPT2 method. The results show the very good performance of the method and its ability to get the potential energy curves for several excited states. The proper convergence of the relativistic infinite order twocomponent IOTC method to the nonrelativistic limit has been demonstrated as well.

Table 8 Spectroscopic constants $\left(R_{e}\right.$ in $\AA ; D_{e}, T_{e}$ in $\mathrm{eV} ; \omega_{e}, \omega_{e} x_{e}$ in $\left.\mathrm{cm}^{-1}\right)$ for the NaLi molecule in the relativistic IOTC CASSCF/CASPT2 and non-relativistic (NR) CASSCF/CASPT2 method using uncontracted POLDK basis set. $1^{1} \Pi, 1^{3} \Pi$ states

\begin{tabular}{llll}
\hline State & & Method & NR CASSCF/CASPT2 \\
& & IOTC CASSCF/CASPT2 & 3.241 \\
\hline $1^{1} \Pi$ & $R_{e}(\AA)$ & 3.242 & 0.182 \\
& $D_{e}(\mathrm{eV})$ & 0.181 & 180.5 \\
& $\omega_{e}\left(\mathrm{~cm}^{-1}\right)$ & 179.2 & 4.8 \\
& $\omega_{e} x_{e}\left(\mathrm{~cm}^{-1}\right)$ & 4.9 & 2.471 \\
$T_{e}(\mathrm{eV})$ & 2.473 & 2.871 \\
$1^{3} \Pi$ & $R_{e}(\AA)$ & 2.870 & 1.176 \\
& $D_{e}\left(\mathrm{eV}^{1}\right)$ & 1.173 & 247.7 \\
& $\omega_{e}\left(\mathrm{~cm}^{-1}\right)$ & 247.8 & 1.2 \\
& $\omega_{e} x_{e}\left(\mathrm{~cm}^{-1}\right)$ & 1.2 & 1.478 \\
& $T_{e}(\mathrm{eV})$ & 1.480 & \\
\hline
\end{tabular}


Funding information This work received support from the National Science Center, Poland (Grant No. 2017/25/B/ST4/01300).

\section{Compliance with ethical standards}

Competing interests The authors declare that they have no competing interests.

Open Access This article is distributed under the terms of the Creative Commons Attribution 4.0 International License (http:// creativecommons.org/licenses/by/4.0/), which permits unrestricted use, distribution, and reproduction in any medium, provided you give appropriate credit to the original author(s) and the source, provide a link to the Creative Commons license, and indicate if changes were made.

\section{References}

1. Balakrishnan N (2016) J Chem Phys 145:150901-1-24

2. Bohn JL, Rey AM, Ye J (1007) Science 357:1002

3. Tajti A, Stanton JF, Matthews DA, Szalay PG (2018) J. Chem Theory Comput 14:5859-5869

4. Stanton JF, Bartlett RJ (1993) J Chem Phys 98:7029-7039

5. Bartlett R (2012) Wiley Interdiscip Rev Comput Mol Sci 2:126138

6. Krylov AI (2008) Annu Rev Phys Chem 59:433-462

7. Musiał M, Lupa Ł, Kucharski SA (2016) J Chem Phys 144:154105-1-9

8. Furche F, Ahlrichs R (2002) J Chem Phys 117:7433-7447

9. Casida ME, Huix-Rotllant M (2012) Annu Rev Phys Chem 63:287-323

10. Szalay PG, Müller T, Gidofalvi G, Liszka H, Shepard R (2012) Chem. Rev. 112:108-181

11. Musiał M, Lupa Ł, Kucharski SA (2017) Mol Phys 115:579-586

12. Liszka H, Nachtigalova D, Aquino AJA, Szalay PG, Plasser F, Machado FBC, Barbatti M (2018) Chem Rev 118:7293-7361

13. Mieszczanin P, Musiał M, Kucharski S (2014) Mol Phys 112:726-732
14. Walczak E, Szefczyk B, Andruniow T (2013) J Chem Theory Comp 9:4915-4927

15. Aquilante F, Bondo-Pederson T, Veryazov V, Lindh R (2013) Wires Comput Mol Sci 3:1430149

16. Mabrouk N, Berriche H (2008) J Phys B At Mol Phys 41:155101

17. Eliav E, Kaldor U (2012) Chem Phys 1:78-82

18. Shee A, Saue T, Visscher L, Gomes ASP (2018) J Chem Phys 149:174113

19. Reiher M, Wolf A (2009) Relativistic quantum chemistry. The Fundamental Theory of Molecular Science, WILEY-VCH Verlag $\mathrm{GmbH}$ and $\mathrm{Co}$. KGaA

20. Iliaš M, Saue T (2007) J Chem Phys 126:064102-1-9

21. Liu J, Cheng L (2018) J Chem Phys 148:144108-1-10

22. Barysz M (2010) In: Barysz M, Ishikawa Y (eds) Relativistic Methods for Chemists. Springer, New York

23. Barysz M (2016) In: leszczyński J, Shukla MK (eds) Practical Aspects of Computational Chemistry IV. Springer Science+Business Media, New York

24. Aquilante F, de Vico L, Ferre N, Ghigo G, Malmqvist PA, Neogrády $\mathrm{P}$, Pedersen TB, Pitoňák M, Reiher $\mathrm{M}$, Roos BO, Serrano-Andres L, Urban M, Veryazov V, Lindh R (2010) J Comput Chem 31:224-247

25. The IOTC method was implemented in the version of the Molcas 7.3 system of programs by Slovakia Group of Quantum Chemistry (Comenius University, Bratislava, Slovakia 2010). The corresponding patches for Molcas 7.3 release can be obtained directly from M. Barysz (e-mail: teomjb@chem.umk.pl) or Vladimir Kellö (e-mail: kelloe@ fns.uniba.sk)

26. Anderson K, Malmqvist PA, Roos BO (1990) J Phys Chem 94:5483-5488

27. Anderson K, Malmqvist PA, Roos BO (1992) J Phys Chem 96:1218-1226

28. Roos BO, Veryazov V, Widmark PO (2003) Theoret Chem Acc 111:345-351

29. Cernusak I, Kellö V, Sadlej AJ (2003) Collect Czech Chem Commun 68:211-239

Publisher's note Springer Nature remains neutral with regard to jurisdictional claims in published maps and institutional affiliations. 